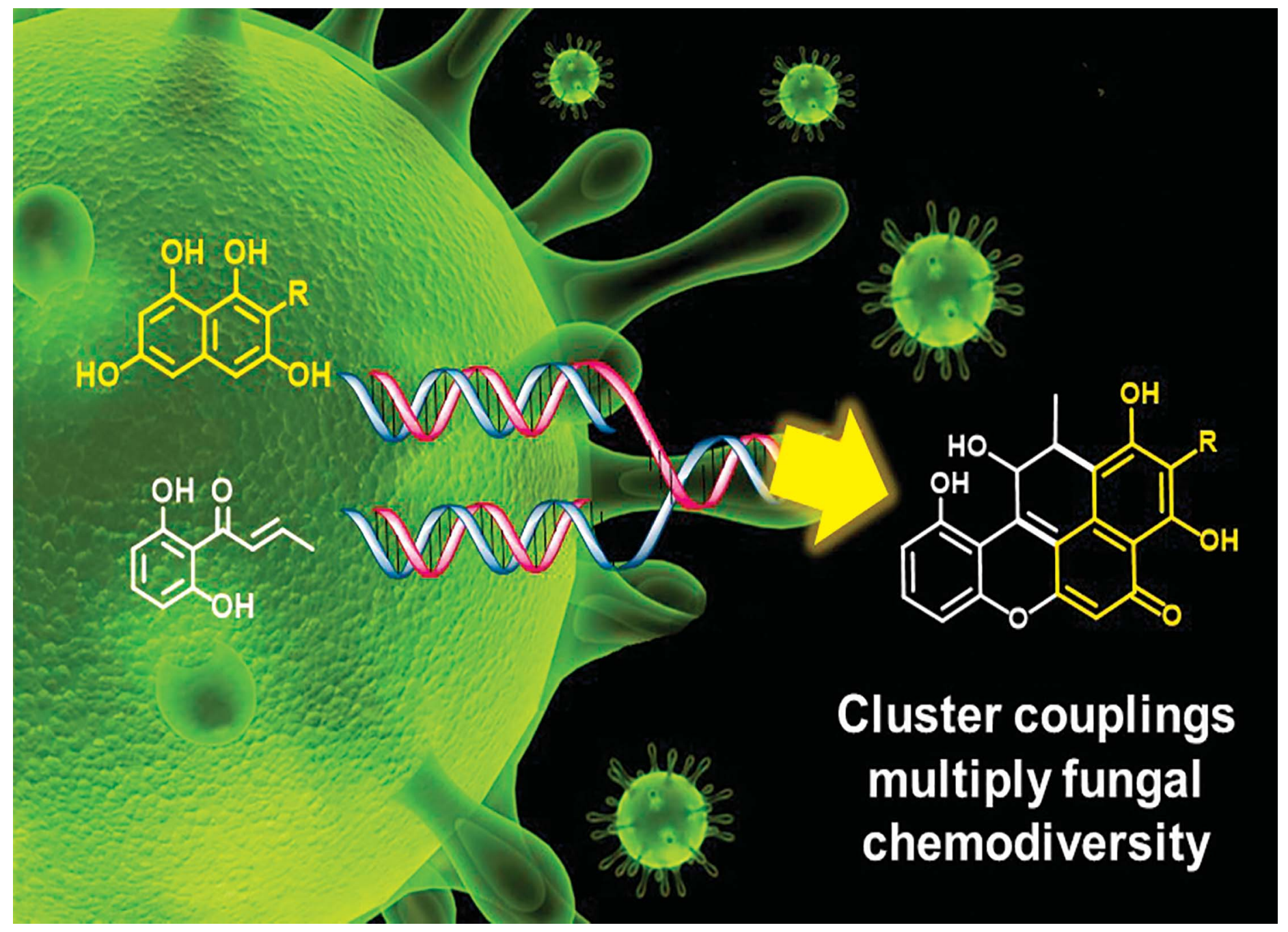

Showcasing research progress from Professor Ren Xiang Tan's laboratories at Nanjing University \& Nanjing University of Chinese Medicine, Nanjing, China.

Dalmanol biosyntheses require coupling of two separate polyketide gene clusters

The biosynthesis of immunosuppressants (dalmanol A and acetodalmanol A) is characterized by the coupling of two separate (naphthalene- and chromane-encoding) gene clusters under the catalysis of unspecific enzyme(s) that may distribute widely in the fungal kingdom. The finding highlights an access to the inter-cluster orchestration derived chemodiversity of microbial secondary metabolites that remain to be a reliable source of leading compounds triggering the development of new medicines, agrochemicals, and other tool molecules.

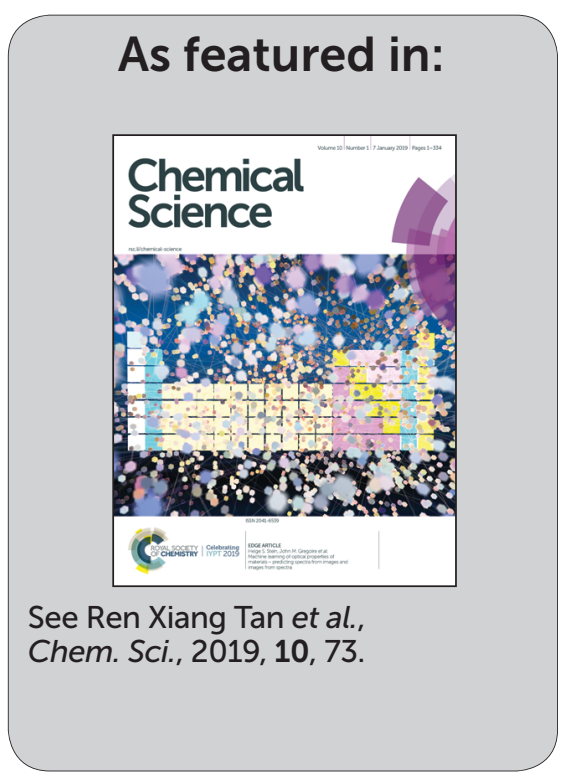


Check for updates

Cite this: Chem. Sci., 2019, 10, 73

¿ All publication charges for this article have been paid for by the Royal Society of Chemistry

\title{
Dalmanol biosyntheses require coupling of two separate polyketide gene clusters $\uparrow$
}

\author{
Zhen Zhen Zhou, ${ }^{a}$ Hong Jie Zhu, ${ }^{a}$ Li Ping Lin, ${ }^{\text {bc }}$ Xuan Zhang, ${ }^{a}$ Hui Ming Ge, (D) ${ }^{a}$ \\ Rui Hua Jiao and Ren Xiang Tan (D) *ab
}

Polyketide-polyketide hybrids are unique natural products with promising bioactivity, but the hybridization processes remain poorly understood. Herein, we present that the biosynthetic pathways of two immunosuppressants, dalmanol $A$ and acetodalmanol $A$, result from an unspecific monooxygenase triggered hybridization of two distinct polyketide (naphthalene and chromane) biosynthetic gene clusters. The orchestration of the functional dimorphism of the polyketide synthase (ChrA) ketoreductase (KR) domain (shortened as ChrA KR) with that of the KR partner (ChrB) in the bioassembly line increases the polyketide diversity and allows the fungal generation of plant chromanes (e.g., noreugenin) and phloroglucinols (e.g., 2,4,6-trihydroxyacetophenone). The simultaneous fungal biosynthesis of 1,3,6,8and 2-acetyl-1,3,6,8-tetrahydroxynaphthalenes was addressed as well. Collectively, the work may symbolize a movement in understanding the multiple-gene-cluster involved natural product biosynthesis, and highlights the possible fungal generations of some chromane- and phloroglucinolbased phytochemicals.

Received 19th August 2018

Accepted 21st November 2018

DOI: $10.1039 / \mathrm{c} 8 \mathrm{sc} 03697 \mathrm{~g}$

rsc.li/chemical-science
In plants, the genes responsible for the phytochemical biosynthesis are usually scattered in different parts to facilitate the efficient organ-based responses to biotic and abiotic stresses, ${ }^{10}$ except for a few gene clusters encoding the specialized metabolic pathways. ${ }^{11}$ In fungi and bacteria, the genes that govern the assembly of a particular secondary metabolite are usually arranged in a cluster. ${ }^{12}$ As an expansion to the "dogma", the biosynthetic gene cluster of polyketides interacts with those of terpenoids to produce meroterpenoids (e.g., austinol and dehydroaustinol) composed typically of polyketide- and isoprenoid-featured substructures. ${ }^{13}$ Inspired by such hybridized forms of SMs, ${ }^{14}$ we were curious about the possible interaction between different polyketide biosynthetic gene clusters, and if existing such inter-cluster couplings may multiply remarkably the skeletal diversity of microbial polyketides. ${ }^{15}$

Dalmanol A (1) and acetodalmanol A (2), sharing a unique 6/ 6/6/6/6 ring system, were obtained as immunosuppressive agents from mantis-associated fungus Daldinia eschscholzii (Fig. 1A). ${ }^{16}$ Our previous ${ }^{16}$ and present attention to its SM profile confirms that the fungus produces, in addition to 1 and 2 , two groups of pentaketides with one and two benzene nuclei. The pentaketides with a benzene ring include 1-(2,6dihydroxyphenyl)butane-1,3-dione (DPB) derived hemiketal (3), 1-(2,6-dihydroxyphenyl)but-2-en-1-one (PBEO, 4), 1-(2,6dihydroxyphenyl)butan-1-one (5), 5-hydroxy-2-methyl-4Hchromen-4-one (6), and 2,3-dihydro-5-hydroxy-2methylchromen-4-one (7) (Fig. 1B). The polyketides with two aromatic nuclei are exemplified by 1,3,6,8-tetrahydroxynaphthalene $(4 \mathrm{HN})$ and acetyl-1,3,6,8-tetrahydroxynaphthalene
${ }^{a}$ State Key Laboratory of Pharmaceutical Biotechnology, Institute of Functional Biomolecules, Nanjing University, Nanjing 210023, China.E-mail: rxtan@nju.edu.cn ${ }^{b}$ State Key Laboratory Cultivation Base for TCM Quality and Efficacy, Nanjing University of Chinese Medicine, Nanjing 210023, China

${ }^{c}$ State Key Laboratory Elemento-Organic Chemistry, Nankai University, Tianjin 300071, China

$\dagger$ Electronic supplementary information (ESI) available. See DOI: $10.1039 / \mathrm{c} 8 \mathrm{sc} 03697 \mathrm{~g}$ 


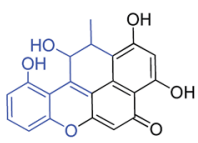

dalmanol A (1)

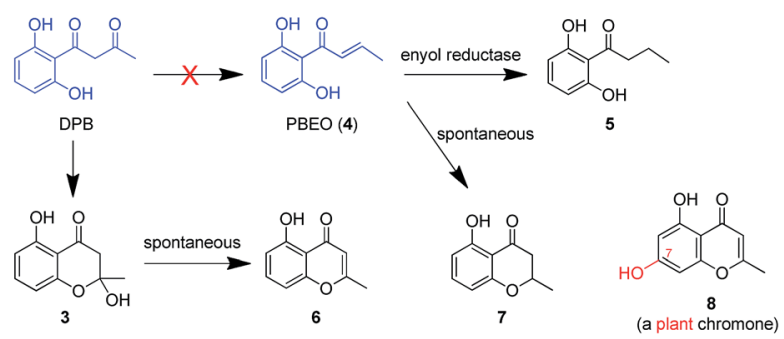

Fig. 1 Representative frameworks of chromane-related polyketides (A) Dalmanol A (1) and acetodalmanol A (2) signify hybridizations between the chromane and naphthalene biosynthetic pathways. (B) The biosynthetic network of fungal pentaketides 5-7 that are skeletally identical with noreugenin (8) from plant (Rheum palmatum). ${ }^{20} \mathrm{DPB}, 1-$ (2,6-dihydroxyphenyl)butane-1,3-dione. PBEO (4), 1-(2,6-dihydroxyphenyl)but-2-en-1-one.

(A4HN). ${ }^{16,17}$ This observation encouraged us to hypothesize that 1 and 2 could be biosynthesized through the hybridization of chromane-related precursor(s) with $4 \mathrm{HN}$ and $\mathrm{A} 4 \mathrm{HN}$, which were also presumed to be involved in the construction of dalmanol $\mathrm{B}$ and acetodalmanol B. ${ }^{\mathbf{1 6}}$ Since no more polyketide synthase (PKS) gene is included in the gene clusters controlling the assembly of $4 \mathrm{HN}^{17}$ and $\mathrm{A} 4 \mathrm{HN},{ }^{18}$ we hypothesized that 1 and 2 could be among the compounds that may showcase the mechanism underlying the coupling of two separate polyketide biosynthetic gene clusters. Here, the intra- and intercellular couplings between the two distinct (naphthalene- and chromane-encoding) polyketide gene clusters is addressed to play a decisive role in the biosynthesis of $\mathbf{1}$ and $\mathbf{2}$ with the crosscluster hybridization most likely triggered by an unspecific monooxygenase found in fungi. In addition to ascertaining the simultaneous fungal biosynthesis of $4 \mathrm{HN}$ and $\mathrm{A} 4 \mathrm{HN}$, the functionally dimorphic orchestration of the ketoreductase (KR) domain and KR partner in the chromane bioassembly line is demonstrated to allow the fungal production of the plant chromones and phloroglucinols.

\section{Results}

\section{Identification of individual gene clusters encoding chromane-} and naphthalene-based polyketides

The investigation was facilitated by our whole-genome sequencing of the $D$. eschscholzii genome while addressing the biosynthetic pathways of dalesconols A-C. ${ }^{17}$ As supposed, copresent in the fungal genome are the genes encoding both partially reducing (PR) and non-reducing (NR) PKSs. In view of the attribution of the NR-PKS to the $4 \mathrm{HN}$ generation, ${ }^{17}$ the PRPKS might be simultaneously involved in the production of $\mathbf{1}$, 2, and 5-7 (Fig. 1), which are abundant enough to be analyzed by the liquid chromatography hyphened with high-resolution mass spectrometry (LC-HR/MS). ${ }^{16}$ Both type III and type I PKSs (including NR-PKS and PR-PKS) have been found to involve in the aromatic polyketide biosynthesis in fungi and plants. ${ }^{19}$ In particular, a type III PKS catalyzes in plants (e.g., Rheum palmatum) the biosynthesis of noreugenin $(8),{ }^{20}$ a 7 hydroxylated derivative of 6 (Fig. 1B). We were prompted to delete first the only type III PKS gene (GME9039) in the $D$. eschscholzii genome. However, such a mutation procedure exerted no effect on the production of 1, 2, and 5-7, as indicated by the LC-HR/MS screening of the EtOAc extract of the mutant culture. Therefore, the type I PKS is required for the assembly of these polyketides. Thus, the gene expression level of all NR- and PR-PKSs (Table S2 $\dagger$ ) in the fungal genome was assessed using quantitative reverse transcription PCR (qRT-PCR). Highly expressed were an NR-PKS (encoded by GME5781) and a PR-PKS (encoded by GME8183) (Fig. S1†). The NR-PKS is pksTL which was demonstrated to catalyze the biosynthesis of naphthalenebased dalesconols A-C. ${ }^{\mathbf{1 7}}$ The PR-PKS encoded by GME8183, hereafter referred to as ChrA, might catalyze the architectural construction of the chromane-based pentaketides such as 5-7. The chromane-encoding gene cluster seems to be widespread in the fungal kingdom since these pentaketides and/or their congeners have been characterized from taxonomically distant fungi such as Daldinia loculata, ${ }^{21}$ Cryptosporiopsis sp. ${ }^{22}$ Phialophora gregata, ${ }^{23}$ and Nodulisporium sp. $^{24}$ To confirm the postulation, the ChrA gene was deleted from the fungal genome to obtain the $\Delta C h r A$ mutant, which was shown to produce none of 1 and 2 as evidenced from the LC-HR/MS screening of the extract derived from its culture (Fig. 2B). Bioinformatic analysis showed that ChrA encodes a type I PR-PKS (ChrA) with 2089 amino acid residues, containing the domains of $\beta$-ketoacyl synthase (KS), acyltransferase (AT), dehydratase (DH), $C$-methyltransferase (MT), ketoreductase (KR), and acyl carrier protein (ACP) (Table S3†). Since $\mathbf{1}$ and $\mathbf{2}$ are non-methylated, the MT domain of ChrA was assumed to be deficient in spite of its possession of the residues (such as D/ExGxGxG) required for $S$ adenosyl-L-methionine (SAM) binding and methylation (Fig. S2 $\dagger$ ). ${ }^{25}$ Such a methyltransferase deficiency was noticed earlier in some eukaryotic cells. ${ }^{26}$ Adjacent to the ChrA gene is $C h r B$, which was found to encode a KR partner. Deletion of $C h r B$ abolished the production of $\mathbf{1}$ and 2. As expected, the naphthol trimers, dalesconols $\mathrm{A}$ and $\mathrm{B}$, became more abundant in the $\Delta C h r A$ and $\Delta C h r B$ mutant cultures (Fig. S15 $\dagger$ ). Next, deletion of the $p k s T L$ gene from the fungal genome as detailed ${ }^{17}$ gave the $\Delta p k s T L$ mutant which was unable to generate $\mathbf{1}$ and $\mathbf{2}$ as well as dalesconols A and B (Fig. 2B and S17 $\dagger$ ). Similarly, more accumulation of chromane-based pentaketides (5-7, 9, and 10) was observed in the $\Delta p k s T L$ mutant (Fig. S16†). Moreover, the formation of 1 was restored by supplementing $4 \mathrm{HN}$ in the $\Delta p k s T L$ mutant culture (Fig. S22 $\dagger$ ). Accordingly, the fungal construction of 1 and 2 requires the co-participation of the naphthalene- and chromane-biosynthesizing gene clusters. These two clusters were found to reside in scaffolds 20 and 36 respectively, and are at least $493 \mathrm{~kb}$ apart even if the two scaffolds anchored most closely on the same chromosome (Fig. 2A and $\mathrm{S} 4 \dagger$ ). 
A

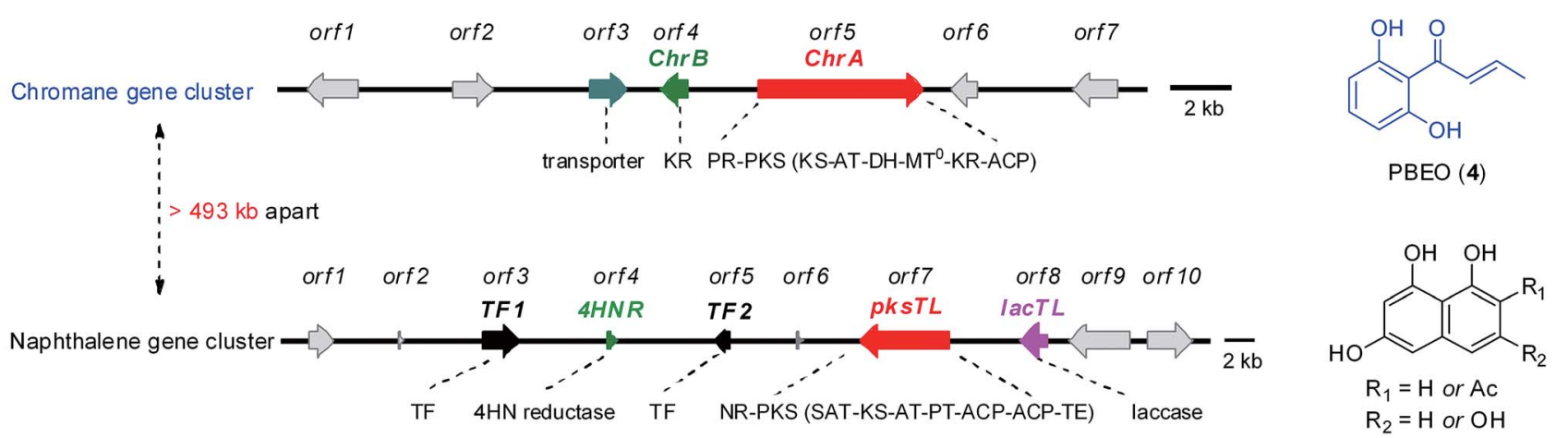

B

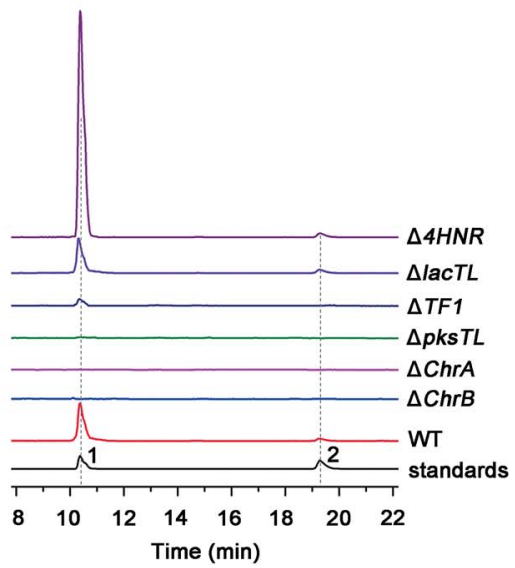

c

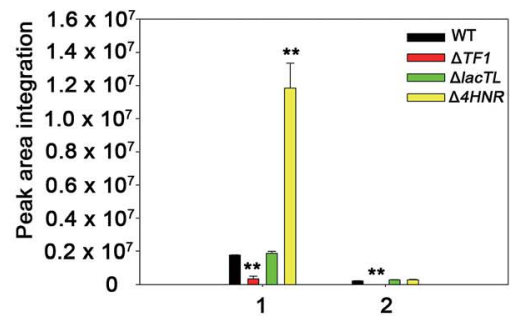

D

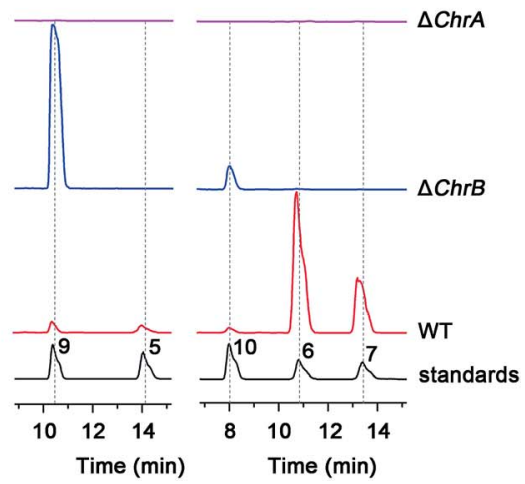

Fig. 2 Polyketide productivity comparison among the wild-type (WT) and mutants of $D$. eschscholzii. (A) The two gene clusters encoding naphthalene and chromane. The WT strain and mutants ( $\triangle$ H HNR, $\Delta$ lacTL, $\Delta T F 1, \Delta p k s T L, \Delta C h r A$, and $\Delta C h r B$ ) were compared through the LC-HR/ MS profiling for the production of: (B) dalmanol A (1) and acetodalmanol A (2) (peak areas integrated (C)) and (D) 5-7, 9 and 10, with authentic samples co-analyzed as references. Data shown as mean $\pm \mathrm{SD}(n=3)$. ** indicated $P<0.01$, by Student's $t$ test.

Simultaneous biosynthesis of 1,3,6,8- (4HN) and 2-acetyl1,3,6,8-tetrahydroxynaphthalenes (A4HN)

The above observation prompted us to hypothesize that the abundance of 1 and 2 could be influenced by the fluctuation in the $4 \mathrm{HN}$ and $\mathrm{A} 4 \mathrm{HN}$ availability. To address the assumption, the $4 \mathrm{HN}$ reductase (4HNR) encoding gene ( $4 \mathrm{HNR}$ ) was knocked out to give the $\triangle 4 H N R$ mutant, which displayed an escalated abundance of $\mathbf{1}$, but with a negligible change in the production of 2 (Fig. 2B and C). Meanwhile, the chromane-based pentaketides such as 6 and 7 was discerned to be less abundant in the $\triangle 4 H N R$ mutant (Fig. S16 $\dagger$ ). Furthermore, deletion of the laccase encoding gene (lacTL) exerted no effect on the abundance of 1 and 2 (Fig. 2B and C). Such mutation results signalled the coinvolvement of the pksTL, ChrA, and $C h r B$ genes in the generation of 1 and 2, while confirming the participation of $4 \mathrm{HNR}$ in the biosynthesis of melanin ${ }^{18}$ and dalesconols A-C (Table S4 $\dagger$ ). ${ }^{17}$ In particular, the $4 H N R$ deletion from the fungal genome results exclusively in the $4 \mathrm{HN}$ boost, which in turn increased the production of 1 , but not 2 . This observation raised our curiosity about the fungal generation of $4 \mathrm{HN}$ and A4HN. Previously, $4 \mathrm{HN}$ was reported to result largely from the A4HN deacetylation through different mechanisms. ${ }^{18,27}$ For example, in Wangiella dermatitidis, $4 \mathrm{HN}$ is produced by deacetylating $\mathrm{A} 4 \mathrm{HN}$ under the catalysis of WdYg1p, a serine hydrolase homolog of Ayg1p. ${ }^{18}$ In
Colletotrichum lagenarium, the deacetylation of A4HN into $4 \mathrm{HN}$ is catalyzed by the bifunctional thioesterase (TE) domain of the PKS, which is simultaneously involved in the cyclization of the enzyme-bound monocyclic hexaketide intermediate. ${ }^{27}$ The TE domain of pksTL in D. eschscholzii bears $46 \%$ and $81 \%$ sequence identity with those of $W$. dermatitidis (WdPKS1) ${ }^{18}$ and C. lagenarium (PKS1) ${ }^{27}$ (Fig. S14 $\dagger$ ).

Moreover, some fungal PKS TE domain could be substratepromiscuous as evidenced both from the simultaneous production of the skeletally alike penta- and hexaketides, ${ }^{\mathbf{1 6 , 2 7}}$ and from the earlier observation that $\mathrm{TE}$ domain is not a determinant of the chain-length specificity of polyketides. ${ }^{28}$ We intuited that there might exist a 'parallel production mechanism' for $4 \mathrm{HN}$ and A4HN, namely, the TE domain of pksTL in $D$. eschscholzii might be able to catalyze in parallel the constructions of $4 \mathrm{HN}$ and $\mathrm{A} 4 \mathrm{HN}$ from the penta- and hexaketide precursors, respectively. Herein, this assumption was reinforced by taking the advantage of the involvement of $4 \mathrm{HN}$ and $\mathrm{A} 4 \mathrm{HN}$ in the biosynthesis of $\mathbf{1}$ and 2, respectively (Fig. 3). The rationalization seems obvious. The $4 H N R$ deletion quenches the reduction of $4 \mathrm{HN}$ into 1,3,8-trihydroxynaphthalene $(3 \mathrm{HN})^{17}$ to generate a ' $4 \mathrm{HN}$ boost'. Such a sharp escalation of the $4 \mathrm{HN}$ level should lead to a clearly increased $\mathrm{A} 4 \mathrm{HN}$ accumulation if $4 \mathrm{HN}$ was formed via the $\mathrm{A} 4 \mathrm{HN}$ deacetylation 


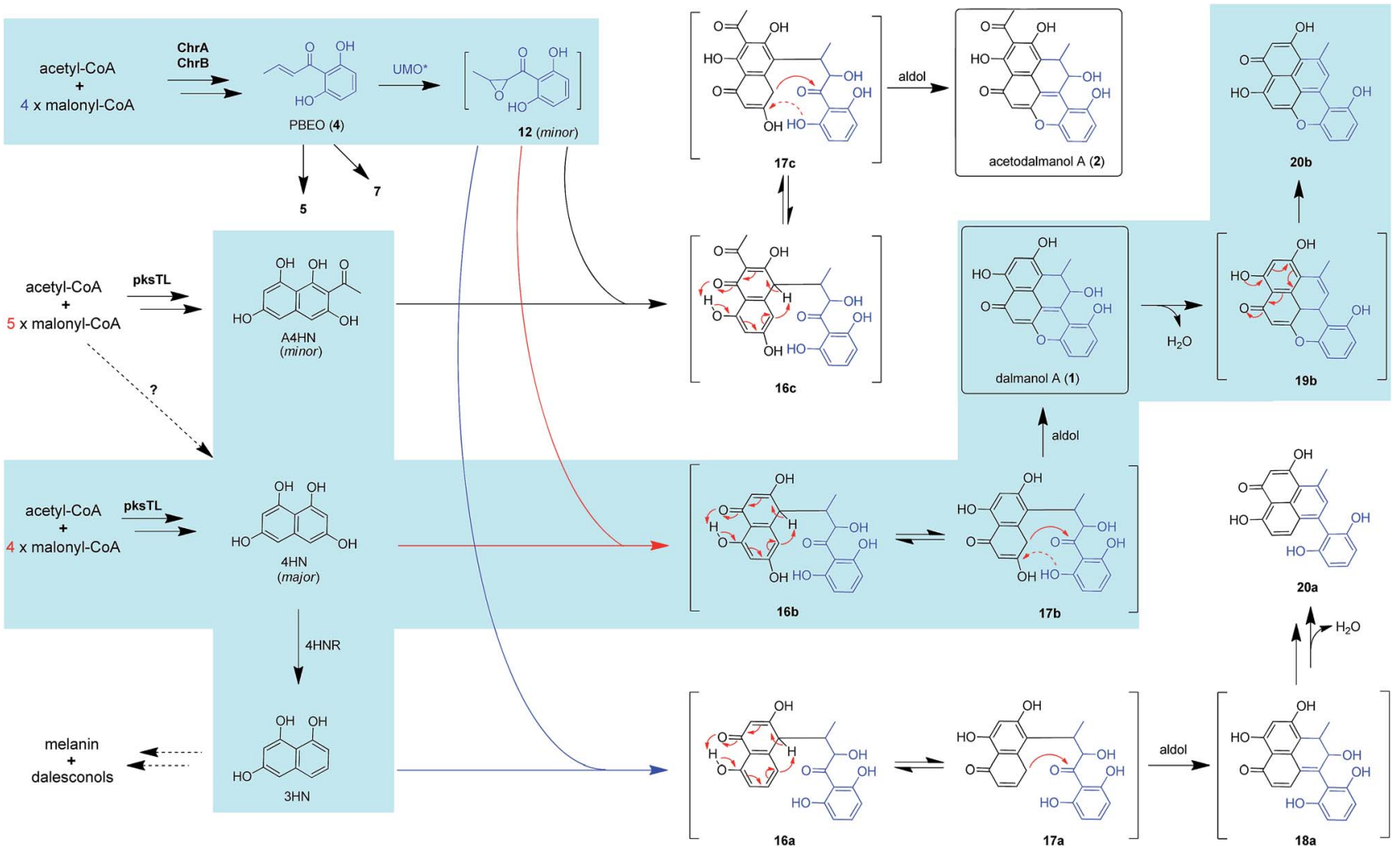

Fig. 3 Proposed biosynthetic pathways for dalmanol A (1) and acetodalmanol A (2). The dalmanol carbon skeleton formation requires the naphthalene and chromane biosynthetic gene clusters, which interact intra- and intercellularly with the presumable participation of an unspecific fungal monooxygenase expressible in D. eschscholzii and A. oryzae. UMO*, unspecific monooxygenase. Dotted and solid red arrows in 17b and 17c indicate the electron transfer in the intramolecular addition and aldol reactions, respectively.

process as reported. ${ }^{18,27}$ However, our deletion of the $4 H N R$ gene enriched 1 only, without substantial effect on the production of 2, suggesting that the A4HN availability was negligibly dependent, or independent, of the $4 \mathrm{HN}$ boost resulting from the $4 H N R$ deletion (Fig. $2 \mathrm{~B}$ and C). This observation could be more convincingly reasoned by the parallel generation of $4 \mathrm{HN}$ and A4HN in D. eschscholzii (Fig. 3), although that the pksTLcatalyzed generation of $4 \mathrm{HN}$ as in case of PKS1 in C. lagenarium $^{27}$ (Fig. 3) could not be definitely ignored.

\section{Functional analysis of transcription factors in naphthalene biosynthetic gene cluster}

Bioinformatic analysis indicated the presence of two transcription factor genes (TF1 and TF2) in the naphthaleneencoding gene cluster (Fig. 2A and Table S4 $\dagger$ ). Deletion of TF1 from the fungal genome significantly reduced the abundance of $\mathbf{1}$ and completely abolished the production of $\mathbf{2}$. More abundant chromane-based pentaketides $(\mathbf{5}-\mathbf{7}, \mathbf{9}$, and $\mathbf{1 0})$ were produced by the $\triangle T F 1$ mutant (Fig. S16 $\dagger$ ). Overexpression of TF1 (TF1 OE) under the control of strong $\alpha$-amylase $(a m y B)$ promoter greatly increased the amount of 1 , but decreased that of 2 (Fig. S18 $\dagger$ ). This observation underpinned that TF1 was an upregulator specific for the naphthalene biosynthetic gene cluster (Fig. 2B and $\mathrm{C}$ ), and that $4 \mathrm{HN}$ and $\mathrm{A} 4 \mathrm{HN}$ might have to compete for the same outside-cluster molecular partner to form $\mathbf{1}$ and 2, respectively. To ascertain whether other naphthols such as $3 \mathrm{HN}$ are involved in the outside-cluster molecule, a large-scale fermentation of the TF1 OE strain was carried out to lead to the characterization of a new minor shunt compound 20a, which could be reasoned to be a hybrid of $3 \mathrm{HN}$ with the outsidecluster pentaketide intermediate (12). Co-existed with 20a was 20b (a dehydrated product of 1, Fig. 3), reinforcing the proposed competition among naphthols for the intermediate 12 (Fig. S56-S65†). The upregulation of naphthalene-encoding gene cluster by TF1 was confirmed by the negligible production of other naphthol-condensed biomolecules including melanin and dalesconols $\mathrm{A}$ and $\mathrm{B}^{16,17}$ by the $\triangle T F 1$ strain of $D$. eschscholzii (Fig S17†). Compared with that of wild type, more abundant chromane-related pentaketides (5-7, 9, and 10) were observed in the $\Delta p k s T L$ or $\triangle T F 1$ mutants (Fig S16 $\dagger$ ). However, the abundance of pentaketides 6 and 7 was reduced upon deleting $4 H N R$, because of the increased availability of $4 \mathrm{HN}$ in the $\triangle 4 H N R$ mutant. Unfortunately, our repeated deletion of TF2 in the gene cluster was unsuccessful.

\section{Heterologous expression of individual gene clusters in Aspergillus oryzae}

To better understand the inter-cluster coupling, we were motivated to identify/confirm the individual gene function in the naphthalene- and chromane-encoding gene clusters. However, 
pksTL and ChrA were unable to be expressed in Escherichia coli. The frustration was overcome by using as an expression host Aspergillus oryzae NSAR1, a quadruple auxotrophic mutant strain $\left(\right.$ niaD $\left.{ }^{-}, s C^{-}, \Delta \arg B, a d e A^{-}\right)$as outlined elsewhere. ${ }^{33}$ As expected, expression of the $p k s T L$ gene in $A$. oryzae yielded $4 \mathrm{HN}$ as a major product (Fig. S19†). Co-expression of $p k s T L$ and $4 H N R$ in $A$. oryzae afforded $3 \mathrm{HN}$, thus confirming the $4 \mathrm{HNR}$ catalyzed $4 \mathrm{HN}$ reduction (Fig. S19†). Next, the ChrA gene was expressed in A. oryzae to produce 9 and 10, both accumulating in the $\Delta C h r B$ mutant of $D$. eschscholzii (Fig. 2D and $\mathrm{S} 20 \dagger$ ). The ChrB gene was expressed alone in A. oryzae, which was unable to form 5 and 7 upon supplementing $\mathbf{9}$ and 10, respectively. Moreover, none of 5-7 was detectable in the co-culture of the transformants of ChrA-AO and ChrB-AO (Fig. S20, S26, and S27†). These data confirmed ChrA as a pentaketide synthase and ChrB as a KR partner capable of directing, at early-stage, the generation of 7deoxygenated pentaketides 5-7 (Fig. 4). With this ascertained, such heterologous expressions were optimized to characterize the nascent intermediates 3 and $\mathbf{4}$ (Fig. S31-S41†). Furthermore, the co-transformant of $C h r A$ and $C h r B(C h r A / C h r B-A O)$ produced 3-7, 9, and 10. This observation suggested the production of 9 and 10 via a shunt path which was in parallel or competition with the ChrB catalyzed production of 3-7 (Fig. 4). Furthermore, the conversion of $\mathbf{3}$ into $\mathbf{6}$, and $\mathbf{4}$ into 7, was shown to be nonenzymatic since such processes were accomplishable in sterile ME medium (Fig. 4, S24 and S25†). The biosynthesis of 10 is catalyzed by ChrA, a type I PKS, which is distinct from the type III PKS involved in the biosynthesis of $\mathbf{8}$ in the plant $R$. palmatum (Fig. S13†). ${ }^{20}$ However, we were frustrated by the formation of 5 and 9 because no enoyl reductase-encoding gene could be found in the chromane biosynthetic gene cluster. This could only be explained by assuming the presence of an unspecific enoyl reductase outside the cluster, ${ }^{28}$ since $\mathbf{5}$ and $\mathbf{9}$ were detected in the cultures of $D$. eschscholzii and A. oryzae (a heterologous expression host used in the study). Such an unspecific enoyl reductase might be present in some, if not all, fungi since 5 was re-isolated from the cultures of other fungi such as Cryptosporiopsis sp., ${ }^{22}$ Phialophora gregata ${ }^{23}$ and Nodulisporium sp. ${ }^{24}$ Moreover, the ChrA KR domain might function as a bifunctional 'gate-keeper' that allowed the generation of 3 (when inactive) or $\mathbf{4}$ (if active), in collaboration with ChrB as a KR partner (Fig. 4). This set of heterologous (co-)expressions pinpointed the metabolic network about pentaketides 3-7 and 9-10 (Fig. 4; pathways II, III, and IV).

\section{Heterologous expression of KR domain mutated ChrA in A. oryzae}

The aforementioned heterologous expression of ChrA in $A$. oryzae afforded a new pentaketide 13, which is spontaneously transformed to 8 (Fig. S21†), a plant chromone. ${ }^{20}$ To understand the catalytic feature of the KR domain of ChrA, three conserved catalytic residues (Lys, Ser, and Tyr) were sequentially mutated as outlined (Fig. S3†) ${ }^{29}$ Analogously, the catalytic residues (Lys, Ser, Tyr, and Asn) in ChrA was identified as described by Yi Tang and co-workers. ${ }^{29}$ The KR-mutated ChrA genes (ChrA-M1 for $\mathrm{K}^{1829} \mathrm{D}$, ChrA-M2 for $\mathrm{S}^{1853} \mathrm{~A}$, and $C h r A-\mathrm{M} 3$ for $\mathrm{Y}^{1866} \mathrm{~A}$ ) were individually expressed in $A$. oryzae to produce, in addition to 10 and 13, the fungal tetraketide 14 (viz., 2,4,6-trihydroxyacetophenone). ${ }^{16}$ In comparison to 13 , an acetyl unit reduction in 14 was noticed, suggesting that such KR mutations could reduce the extension cycle specificity of ChrA. This phenomenon was discerned when addressing terrein biosynthesis in Aspergillus terreus. ${ }^{28}$ This observation was substantiated by the production of 14 upon co-expressions of $C h r B$ with original and KR-mutated ChrA genes (Fig. 5, traces vi-ix). However, such coexpressions generated four additional peaks, three of which were shown to arise from 3, 6, and 7 (Fig. 5, traces vii-ix). The fourth peak was identified to be 15, which is an adduct of 4 with 3-mercaptolactic acid (a described fungal metabolite) formed via an outlined pathway (Fig. 4, pathway IV). ${ }^{30}$ The above observations could only be explained by the functional dimorphism (active and silent states) of ChrA KR domain that serves as a gate-keeper to diversify the chromane-based polyketides (Fig. 4). It is noteworthy that the KR mutations mitigated, but not abolished as discerned, ${ }^{29}$ the function of the fungal ChrA.

\section{Coupling between chromane- and naphthalene-encoding gene clusters}

Scrutiny of the structural feature of $\mathbf{1}$ and 2 prompted us to hypothesize that the PBEO (4) epoxidation might be required for the coupling between the naphthalene and chromane biosynthetic gene clusters. The stability of $\mathbf{4}$ was examined indicating that it is relatively stable in acetone and unable to be oxidized with air exposure, and tends to form 7 in $\mathrm{MeOH}$, DMSO, and the culture medium (as trapped by xenobiotic indoles ${ }^{31}$ ) (Fig. S25 $\dagger$ ). With the rationalization, 4 was likely epoxidized into 12 under a monooxygenase catalysis, followed by independent couplings with $4 \mathrm{HN}$ and $\mathrm{A} 4 \mathrm{HN}$ to give 1 and 2, respectively (Fig. 3). To address the assumption, ChrA and $4 H N R$ were co-deleted from the D. eschscholzii genome to afford the $\triangle C h r A / \triangle 4 H N R$ mutant, which was reasoned to boost a $4 \mathrm{HN}$ accumulation without producing $\mathbf{3}$ and $\mathbf{4}$ (see above). As expected, the generation of $\mathbf{1}$ by the $\triangle C h r A / \triangle 4 H N R$ mutant was restored by addition of 4 , but unrestorable by re-supplementing 3 , suggesting that 4 and $4 \mathrm{HN}$ were precursors of $\mathbf{1}$ (Fig. S23†). The production of $\mathbf{2}$ failed to be restored by the experiment because $\mathrm{A} 4 \mathrm{HN}$ is much less abundant and nearly independent of the $4 \mathrm{HN}$ boost (see above). By getting the two on-pathway intermediates in hand, 4 and $4 \mathrm{HN}$ were incubated together in ME medium used for culturing $D$. eschscholzii to see if spontaneous coupling could take place. However, 4 rapidly converted to 7 at $5 \mathrm{~min}$, and completely converted to 7 at $30 \mathrm{~min}$ after co-incubation, but no coupling product was detected (Fig. S28†). These results showed that 4 and $4 \mathrm{HN}$ could not spontaneously coupled to generate 1 . Furthermore, no monooxygenase-encoding gene was found within the chromane- and naphthalene-encoding gene clusters. The enzyme catalyzing the PBEO (4) epoxidation could be outside the clusters in the light of the alkene epoxidation catalyzed by cytochrome $\mathrm{P} 450$ monooxygenase ${ }^{32,33}$ or flavincontaining monooxygenase (FMO).$^{34,35}$ Owing to its analogy to styrene, 4 could be epoxidized under the catalysis of styrene monooxygenase, an FMO found in bacteria. ${ }^{36}$ However, our 


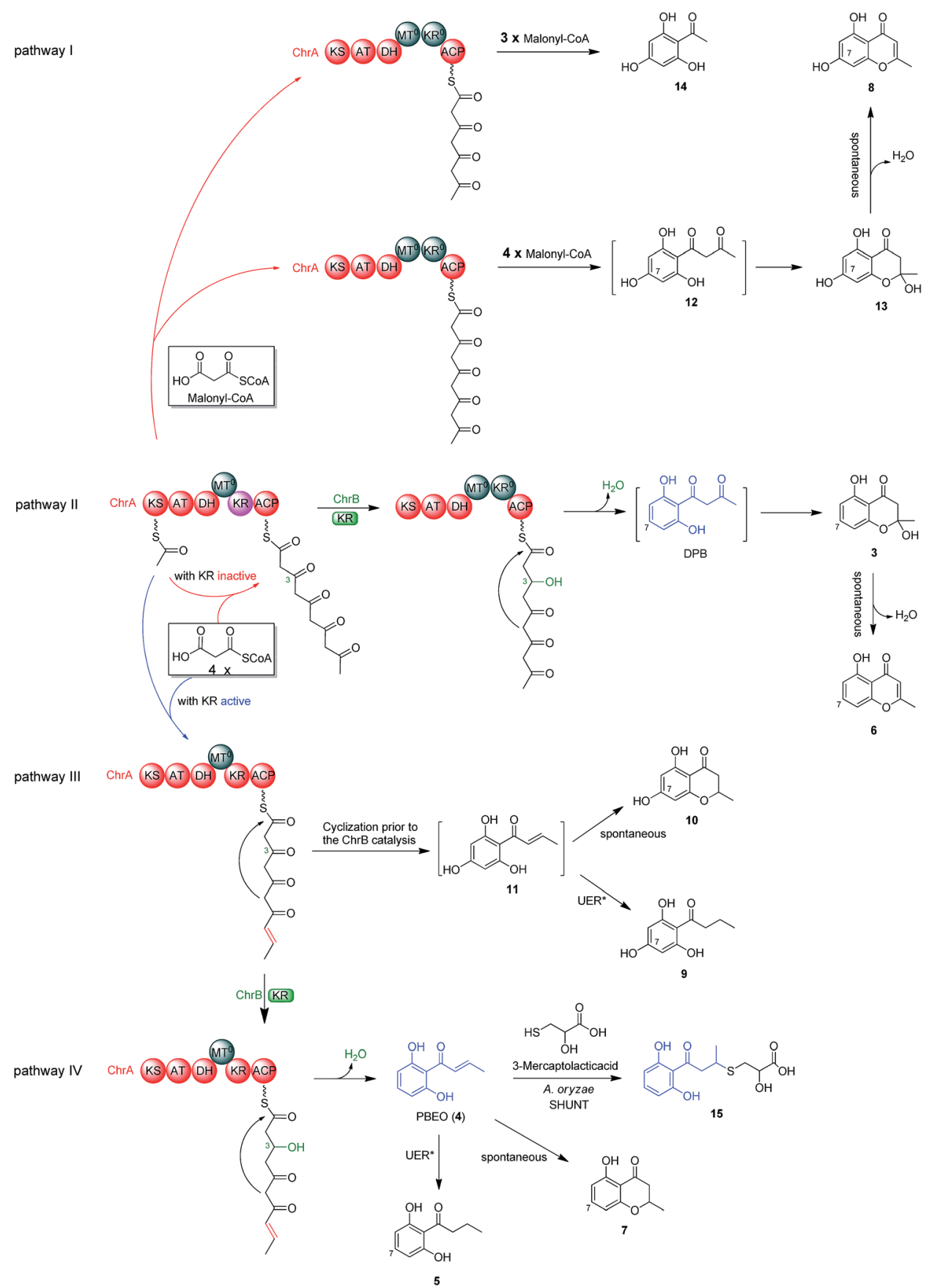

Fig. 4 Metabolic network of polyketides encoded by the chromane gene cluster in D. eschscholzii. ChrA is a partially reducing polyketide synthase (PR-PKS) with the domains of $\beta$-ketoacylsynthase (KS), acyltransferase (AT), dehydratase (DH), C-methyltransferase (MT ${ }^{0}$, inactive), ketoreductase (KR, active/inactive) and acyl carrier protein (ACP). ChrB is a ketoreductase partner that catalyzes the selective 3-ketone reduction of acyclic pentaketide with acetonyl or allyl side-chain terminals. UER*, unspecific enoyl reductase expressible in $D$. eschscholzii and $A$. oryzae.

bioinformatic analysis showed the absence of any bacterial styrene monooxygenase in the fungus (Fig. S29†). Previously, a fungal P450 monooxygenase was evidenced to catalyze the styrene metabolism initialized with the epoxidation of vinyl side-chain. ${ }^{37}$ In the D. eschscholzii genome, ${ }^{17}$ a total of 119 cytochrome P450s and 79 FMOs have been annotated (Table $\left.\mathrm{S} 10^{\dagger}\right)$. Since it is too tedious to identify the epoxidationcatalyzing enzyme of the fungus, we alternatively ascertained the epoxidase type via a gene-implied enzyme inhibition strategy. ${ }^{38}$ Thus, an FMO (methimazole) and two P450 inhibitors (phenylbutazone and proadifen) were supplemented separately in the fungal cultures. As a result, the fungal exposure to the P450 inhibitors substantially reduced the production of 1 and 2 whereas methimazole exerted negligible effect (Fig. S30 $\dagger$ ). Accordingly, the P450, but not FMO, monooxygenase(s) most likely catalyzed the epoxidation of $\mathbf{4}$ in the fungus. 


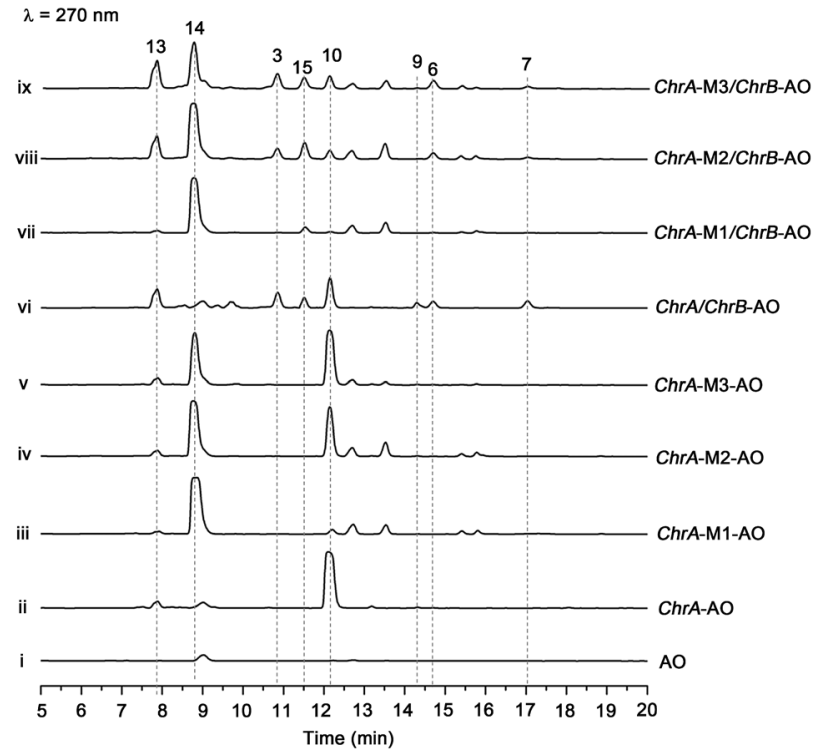

Fig. 5 Co-expression of KR-mutated ChrA and ChrB in A. oryzae (AO) HPLC traces for metabolites produced by $A O$ ((i), as a blank reference), the transformants [with ChrA (ii), ChrA-M1 for K ${ }^{1829} \mathrm{D}$ (iii), ChrA-M2 for $\mathrm{S}^{1853} \mathrm{~A}$ (iv), and ChrA-M3 for $\left.\mathrm{Y}^{1866} \mathrm{~A}(\mathrm{v})\right]$, and the co-transformants with ChrA and ChrB (vi), ChrA-M1 and ChrB (vii), ChrA-M2 and ChrB (viii), and ChrA-M3 and ChrB (ix).

To confirm the assumption above, the two naphthalene and chromane biosynthetic gene clusters were co-expressed in $A$. oryzae. Gratifyingly, $\mathbf{1}$ and $\mathbf{2}$ were produced by the cotransformant with the pksTL, ChrA, and ChrB genes ( $p k s T L /$ ChrA/ChrB-AO) (Fig. 6). These results indicated that the monooxygenase catalyzing the formation of $\mathbf{1 2}$ from $\mathbf{4}$ could be unspecific since expressible in both $A$. oryzae and $D$.

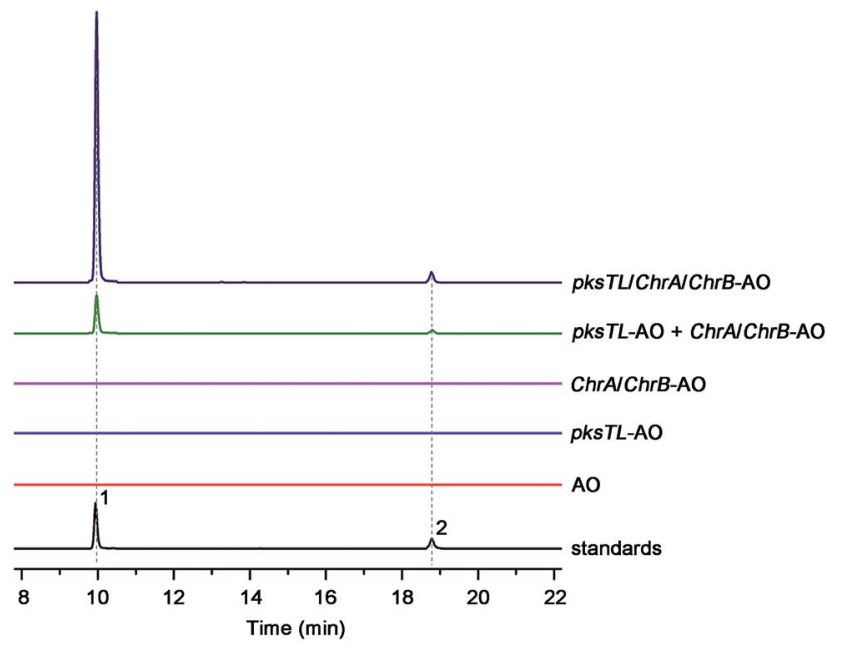

Fig. 6 Production of 1 and 2 via heterologous co-expression and cocultivation. Co-expression of pksTL, ChrA, and ChrB in A. oryzae gave a co-transformant (pksTL/ChrA/ChrB-AO) capable of producing 1 and 2, which were afforded as well by co-culturing the transformant (pksTL-AO) with the co-transformant (ChrA/ChrB-AO). However, none of 1 and 2 was generated if culturing separately the pksTL-AO transformant and the ChrA/ChrB-AO co-transformant. eschscholzii. ${ }^{28}$ Accordingly, 1 and 2 result likely from the monooxygenase-triggered coupling between the naphthalene and chromane biosynthetic gene clusters. Moreover, the involvement of the unspecific enzyme in the inter-cluster coupling strengthened our curiosity about whether the naphthalene and chromane biosynthetic gene clusters would interact if located in different fungal cells. To address the hypothesis, the pksTL-AO transformant was co-cultured with the co-transformant with ChrA/ChrB-AO. As expected, 1 and 2 were formed upon the co-cultivation of these (co-)transformants (Fig. 6). This experimentation highlighted that the two polyketide gene clusters can interact intercellularly, too. The finding may be of considerable value in the rational design of the microbial co-culture approach which has been accepted as a promising access to the improved secondary metabolite chemodiversity for drug discovery. ${ }^{39}$

\section{Discussion}

As versatile biosynthetic precursors of natural products ${ }^{\mathbf{1 6}, 40}$ and virulence factors such as melanin, ${ }^{18} 4 \mathrm{HN}$ and/or $\mathrm{A} 4 \mathrm{HN}$ are widespread polyketides in fungi (this work and many others) with the former producible in bacteria. ${ }^{\mathbf{4 1 , 4 2}}$ Chromane-based polyketides have been characterized as the SMs from diverse fungi ${ }^{16,21-24}$ and plants. ${ }^{20,43}$ Also found are the natural hybrids of chromone with piperidine ${ }^{43}$ and of $4 \mathrm{HN}$ with chromane $\mathrm{e}^{\mathbf{1 6 , 2 4}}$ or isoprenoid motifs. ${ }^{40}$ Therefore, the fresh portion of small molecule chemical space could be occupied by SMs biosynthesizable through the 'collaboration' of multiple gene clusters. This rationalization has been reinforced herein by the elucidated biosynthesis of dalmanol A (1) and acetodalmanol A (2), which reveals the intra- and intercellular hetero-coupling between the naphthalene- and chromane-encoding gene clusters. The cross-cluster coupling manner underlying the formation of $\mathbf{1}$ and $\mathbf{2}$ is different from the meroterpenoid bioassembly (co-supported by the polyketide and terpenoid biosynthetic gene clusters), ${ }^{13}$ and within-cluster PKS pairings. ${ }^{\mathbf{4 4 , 4 5}}$

The cross-cluster coupling could be either spontaneous or enzymatic. Thus, PBEO (4) and $4 \mathrm{HN}$ were incubated together in media used for the fungal culture. To our surprise, 4 became 7 rather than reacted with $4 \mathrm{HN}$ (Fig. S28 $\dagger$ ). Thus, such a unique coupling could only be triggered by an unspecific monooxygenase found in some (if not all) fungi such as D. eschscholzii and $A$. oryzae. ${ }^{28}$ Such a monooxygenase is outside the two polyketide biosynthetic gene clusters and catalyzes the conversion of pentaketide precursor PBEO (4) to the epoxide intermediate 12 which is too labile or less abundant to be identified in the work. The difficulty in characterizing 12 might have been intensified by the competitive conversion of 4 to 7 and 5 via the non-enzymatic intramolecular addition and the reduction catalyzed by an unspecific enoyl reductase expressible in $D$. eschscholzii and A. oryzae (Fig. 4, pathway IV). Similarly, an unspecific ketoreductase was found to involve in the terrein biosynthesis. ${ }^{28}$ This rationalization agrees with the oxygenation pattern of 1 and 2 and the monooxygenase-catalyzed 2,3-epoxidation of some indole motifs (a mimic of the polarized double bond of 4, Fig. 3) in the fungal culture. ${ }^{46}$ The inter-cluster 
coupling gains reinforcements from the production of $\mathbf{1}$ and $\mathbf{2}$ via the heterologous co-expression of pksTL, ChrA and ChrB in $A$. oryzae, and through the co-culture of the two (co-)transformants, $p k s T L-\mathrm{AO}$ and $C h r A / C h r B-A O$. The finding seems to be different from the post-biosynthesis coupling between smallmolecule phenols via a non-enzymatic nucleophilic addition reaction $^{47}$ and the phenolic radical couplings catalyzed by laccase, ${ }^{17}$ multicopper oxidase, ${ }^{48}$ and the within-cluster $\mathrm{P} 450$ enzymes. ${ }^{49,50}$ Interestingly, the addressed coupling between the naphthalene and chromane biosynthetic gene clusters enabled us to identify the simultaneous fungal biosynthesis of $4 \mathrm{HN}$ and A4HN. Along with the transformant co-culture protocol, the driving force for the cross-cluster coupling may help to rationally design and efficiently accomplish the generation of new SMs via microbial co-cultivation approaches.

The chromane-based polyketides are found in fungi (this work and others) and plants such as Aloe arborescens (family Aloaceae), ${ }^{51}$ Dysoxylum acutangulum (Meliaceae), ${ }^{43}$ Eugenia caryophyllata (Myrtaceae), ${ }^{52}$ Crossosoma bigelovii (Crossosomataceae), ${ }^{53}$ Knoxia corymbosa (Rubiaceae), ${ }^{54}$ and Schumanniophyton magnificum (Rubiaceae). ${ }^{55}$ Many chromane derivatives have promising biological activities. ${ }^{56}$ This work highlights the biosynthetic relationship of this family of SMs by genetic manipulation. Interestingly, both ChrA ketoreductase domain (ChrA KR) and ChrB as a KR partner in the same cluster are functionally dimorphic; namely, they exert or lose their catalytic activity during the assembly of chromane polyketides (Fig. 4). Such a KR dimorphism orchestration of ChrA KR and ChrB allows the generation of diversified chromane polyketides (Fig. 4). Previously, the KR domain dimorphism of polyketide synthases was believed to likely arise from a couple of mechanisms such as the module skipping ${ }^{57}$ and chain-length control. ${ }^{58,59}$ Our mutation confirmed that the KR domain affects some polyketide chain-length. In particular, the replacement of Lys ${ }^{1829}$ with Asp residue in ChrA KR afforded an remarkably escalated level of $2^{\prime}, 4^{\prime}, 6^{\prime}$-trihydroxyacetophenone (14), an anti-obesity phytochemical ${ }^{60}$ that serves as a (bio) synthetic starter motif for arrays of bioactive phloroglucinols. $^{61,62}$ More interestingly, when ChrA KR and ChrB both lose the catalytic activity, the fungal chromane biosynthetic pathway constructs 13 that tends to form noreugenin (8), a plant chromone used as a common starting material for the synthesis of bioactive chromone natural products such as ptaeroxylin and eranthin. ${ }^{63}$ These findings suggest that the fungal chassis with such a useful and operable chromane biosynthetic gene cluster could be a proper host for the synthetic biology approaches to chromane- and phloroglucinolbased bioactive phytochemicals and/or precursors thereof.

\section{Conclusions}

We have addressed the two-gene-cluster based biosynthetic pathways of dalmanol A (1) and acetodalmanol A (2). Their unique carbon skeletons form via the monooxygenase triggered cascade reactions between a pair of polyketides constructed by two distinct polyketide (naphthalene and chromane) biosynthetic gene clusters. Such a cross-cluster interaction allowed the identification of the simultaneous fungal biosynthesis of $4 \mathrm{HN}$ and $\mathrm{A} 4 \mathrm{HN}$. A combination of transformant (co-)cultivations with gene deletions and heterologous (co-)expressions disclosed the metabolic network about pentaketides 3-7, 9, and 10, which are produced via the competitive shunt pathways switched likely by the functional dimorphism of ChrA KR and/or the nascent intermediate aromatization before the ChrB catalyzed cyclization (Fig. 4). In the chassis of D. eschscholzii and probably other chromane-synthesizing fungi, ${ }^{21-24}$ the ChrA with an inactivated KR domain seems to be of significance in establishing the synthetic biology approaches to the chromane- and phloroglucinol-based phytochemicals. Collectively, the work sheds light on the understanding of the multiple-gene-cluster based natural product biosynthesis, which is valuable for generating skeletally related phytochemicals and other small molecules with new chemical and biological space.

\section{Conflicts of interest}

There are no conflicts to declare.

\section{Acknowledgements}

The work was co-financed by the NSFC (81530089, 21672101 and 21702099) and MOST grants (2018ZX09711001-007-004). We thank Professor Katsuhiko Kitamoto (University of Tokyo) for providing fungal heterologous expression host (A. oryzae NSAR1) and plasmids (pTAex3, pUSA and pAdeA).

\section{References}

1 S. W. Drew and A. L. Demain, Annu. Rev. Microbiol., 1977, 31, 343-356.

2 N. P. Keller, Nat. Chem. Biol., 2015, 11, 671-677.

3 T. Hautbergue, E. L. Jamin, L. Debrauwer, O. Puel and I. P. Oswald, Nat. Prod. Rep., 2018, 35, 147-173.

4 J. Hubert, J. M. Nuzillard and J. H. Renault, Phytochem. Rev., 2017, 16, 55-95.

5 M. C. Leal, C. Sheridan, R. Osinga, G. Dionisio, R. J. M. Rocha, B. Silva, R. Rosa and R. Calado, Mar. Drugs, 2014, 12, 3929-3952.

6 A. Boufridi and R. J. Quinn, Annu. Rev. Pharmacol., 2018, 58, 451-470.

7 M. J. Smanski, H. Zhou, J. Claesen, B. Shen, M. A. Fischbach and C. A. Voigt, Nat. Rev. Microbiol., 2016, 14, 135-149.

8 X. N. Liu, W. T. Ding and H. F. Jiang, Microb. Cell Fact., 2017, 16, 125.

9 P. Chettri, P. Y. Dupont and R. E. Bradshaw, Mol. Microbiol., 2018, 107, 508-522.

10 J. K. Weng, New Phytol., 2014, 201, 1141-1149.

11 J. K. Weng, R. N. Philippe and J. P. Noel, Science, 2012, 336, 1667-1670.

12 Y. F. Li, K. J. S. Tsai, C. J. B. Harvey, J. J. Li, B. E. Ary, E. E. Berlew, B. L. Boehman, D. M. Findley, A. G. Friant, C. A. Gardner, M. P. Gould, J. H. Ha, B. K. Lilley, E. L. McKinstry, S. Nawal, R. C. Parry, K. W. Rothchild, S. D. Silbert, M. D. Tentilucci, A. M. Thurston, R. B. Wai, 
Y. J. Yoon, R. S. Aiyar, M. H. Medema, M. E. Hillenmeyer and L. K. Charkoudian, Fungal Genet. Biol., 2016, 89, 18-28.

13 H. C. Lo, R. Entwistle, C. J. Guo, M. Ahuja, E. Szewczyk, J. H. Hung, Y. M. Chiang, B. R. Oakley and C. C. C. Wang, J. Am. Chem. Soc., 2012, 134, 4709-4720.

14 Y. Matsuda and I. Abe, Nat. Prod. Rep., 2016, 33, 26-53.

15 W. Cai and W. Zhang, Curr. Opin. Biotechnol., 2017, 50, 3238.

16 Y. L. Zhang, J. Zhang, N. Jiang, Y. H. Lu, L. Wang, S. H. Xu, W. Wang, G. F. Zhang, Q. Xu, H. M. Ge, J. Ma, Y. C. Song and R. X. Tan, J. Am. Chem. Soc., 2011, 133, 5931-5940.

17 W. Fang, S. Ji, N. Jiang, W. Wang, G. Y. Zhao, S. Zhang, H. M. Ge, Q. Xu, A. H. Zhang, Y. L. Zhang, Y. C. Song, J. Zhang and R. X. Tan, Nat. Commun., 2012, 3, 1039.

18 M. H. Wheeler, D. Abramczyk, L. S. Puckhaber, M. Naruse, Y. Ebizuka, I. Fujii and P. J. Szaniszlo, Eukaryotic Cell, 2008, 7, 1699-1711.

19 C. Hertweck, Angew. Chem., Int. Ed., 2009, 48, 4688-4716.

20 I. Abe, Y. Utsumi, S. Oguro, H. Morita, Y. Sano and H. Noguchi, J. Am. Chem. Soc., 2005, 127, 1362-1363.

21 A. K. Nadeau and J. L. Sorensen, Tetrahedron Lett., 2011, 52, 1697-1699.

22 M. K. Zilla, M. Qadri, A. S. Pathania, G. A. Strobel, Y. Nalli, S. Kumar, S. K. Guru, S. Bhushan, S. K. Singh, R. A. Vishwakarma, S. Riyaz-Ul-Hassan and A. Ali, Phytochemistry, 2013, 95, 291-297.

23 L. E. Gray, H. W. Gardner, D. Weisleder and M. Leib, Phytochemistry, 1999, 50, 1337-1340.

24 J. Q. Dai, K. Krohn, S. Draeger and B. Schulz, Eur. J. Org. Chem., 2009, 1564-1569.

25 J. Kennedy, K. Auclair, S. G. Kendrew, C. Park, J. C. Vederas and C. R. Hutchinson, Science, 1999, 284, 1368-1372.

26 A. Dong, J. A. Yoder, X. Zhang, L. Zhou, T. H. Bestor and X. Cheng, Nucleic Acids Res., 2001, 29, 439-448.

27 A. L. Vagstad, E. A. Hill, J. W. Labonte and C. A. Townsend, Chem. Biol., 2012, 19, 1525-1534.

28 C. Zaehle, M. Gressler, E. Shelest, E. Geib, C. Hertweck and M. Brock, Chem. Biol., 2014, 21, 719-731.

29 H. Zhou, Z. Z. Gao, K. J. Qiao, J. J. Wang, J. C. Vederas and Y. Tang, Nat. Chem. Biol., 2012, 8, 331-333.

30 E. Adelin, M. T. Martin, M. F. Bricot, S. Cortial, P. Retailleau and J. Ouazzani, Phytochemistry, 2012, 84, 135-140.

31 L. P. Lin, P. Yuan, N. Jiang, Y. N. Mei, W. J. Zhang, H. M. Wu, A. H. Zhang, J. M. Cao, Z. X. Xiong, Y. Lu and R. X. Tan, Org. Lett., 2015, 17, 2610-2613.

32 M. Schrewe, M. K. Julsing, B. Buhler and A. Schmid, Chem. Soc. Rev., 2013, 42, 6346-6377.

33 F. H. Jin, J. Maruyama, P. R. Juvvadi, M. Akioka and K. Kitamoto, FEMS Microbiol. Lett., 2004, 239, 79-85.

34 A. Minami, M. Shimaya, G. Suzuki, A. Migita, S. S. Shinde, K. Sato, K. Watanabe, T. Tamura, H. Oguri and H. Oikawa, J. Am. Chem. Soc., 2012, 134, 7246-7249.

35 K. C. Van de Bittner, M. J. Nicholson, L. Y. Bustamante, S. A. Kessans, A. Ram, C. J. van Dolleweerd, B. Scott and E. J. Parker, J. Am. Chem. Soc., 2018, 140, 582-585.
36 T. Heine, A. Scholtissek, A. H. Westphal, W. J. H. van Berkel and D. Tischler, Biochim. Biophys. Acta, 2017, 1865, 17701780.

37 H. H. Cox, B. W. Faber, W. N. Van Heiningen, H. Radhoe, H. J. Doddema and W. Harder, Appl. Environ. Microbiol., 1996, 62, 1471-1474.

38 W. Yan, H. M. Ge, G. Wang, N. Jiang, Y. N. Mei, R. Jiang, S. J. Li, C. J. Chen, R. H. Jiao, Q. Xu, S. W. Ng and R. X. Tan, Proc. Natl. Acad. Sci. U. S. A., 2014, 111, 1813818143.

39 S. Bertrand, N. Bohni, S. Schnee, O. Schumpp, K. Gindro and J. L. Wolfender, Biotechnol. Adv., 2014, 32, 1180-1204.

40 L. Kaysser, P. Bernhardt, S. J. Nam, S. Loesgen, J. G. Ruby, P. Skewes-Cox, P. R. Jensen, W. Fenical and B. S. Moore, J. Am. Chem. Soc., 2012, 134, 11988-11991.

41 N. Funa, Y. Ohnishi, I. Fujii, M. Shibuya, Y. Ebizuka and S. Horinouchi, Nature, 1999, 400, 897-899.

42 Y. Sone, S. Nakamura, M. Sasaki, F. Hasebe, S. Y. Kim and N. Funa, Appl. Environ. Microbiol., 2018, 84, e00258-18.

43 P. Klausmeyer, Q. Zhou, D. A. Scudiero, B. Uranchimeg, G. Melillo, J. H. Cardellina, R. H. Shoemaker, C. J. Chang and T. G. McCloud, J. Nat. Prod., 2009, 72, 1879-1883.

44 Y. H. Chooi and Y. Tang, J. Org. Chem., 2012, 77, 9933-9953. 45 M. Sato, J. E. Dander, C. Sato, Y. S. Hung, S. S. Gao, M. C. Tang, L. Hang, J. M. Winter, N. K. Garg, K. Watanabe and Y. Tang, J. Am. Chem. Soc., 2017, 139, 5317-5320.

46 L. P. Lin and R. X. Tan, Chin. J. Chem., 2018, 36, 749-753.

47 C. Huang, C. Yang, W. Zhang, L. Zhang, B. C. De, Y. Zhu, X. Jiang, C. Fang, Q. Zhang, C. S. Yuan, H. W. Liu and C. Zhang, Nat. Commun., 2018, 9, 2088.

48 M. Kawaguchi, T. Ohshiro, M. Toyoda, S. Ohte, J. Inokoshi, I. Fujii and H. Tomoda, Angew. Chem., Int. Ed., 2018, 57, 5115-5119.

49 S. S. Gao, T. Zhang, M. Garcia-Borras, Y. S. Hung, J. M. Billingsley, K. N. Houk, Y. Hu and Y. Tang, J. Am. Chem. Soc., 2018, 140, 6991-6997.

50 L. S. Mazzaferro, W. Huttel, A. Fries and M. Muller, J. Am. Chem. Soc., 2015, 137, 12289-12295.

51 Y. Mizuuchi, S. P. Shi, K. Wanibuchi, A. Kojima, H. Morita, H. Noguchi and I. Abe, FEBS J., 2009, 276, 2391-2401.

52 Y. W. Zhang and Y. W. Chen, Phytochemistry, 1997, 45, 401403.

53 P. Klausmeyer, Q. Zhou, D. A. Scudiero, B. Uranchimeg, G. Melillo, J. H. Cardellina, R. H. Shoemaker, C. J. Chang and T. G. McCloud, J. Nat. Prod., 2009, 72, 805-812.

54 Y. B. Wang, R. Huang, H. B. Zhang and L. Li, J. Asian Nat. Prod. Res., 2006, 8, 663-670.

55 P. Tane, J. F. Ayafor, B. L. Sondengam and J. D. Connolly, Phytochemistry, 1990, 29, 1004-1007.

56 F. M. Awadallah, T. A. El-Waei, M. M. Hanna, S. E. Abbas, M. Ceruso, B. E. Oz, O. O. Guler and C. T. Supuran, Eur. J. Med. Chem., 2015, 96, 425-435.

57 S. C. Wenzel, B. Kunze, G. Hofle, B. Silakowski, M. Scharfe, H. Blocker and R. Muller, ChemBioChem, 2005, 6, 375-385.

58 X. L. Chen, Z. F. Guo, P. M. Lai, K. H. Sze and Z. H. Guo, Angew. Chem., Int. Ed., 2010, 49, 7926-7928. 
59 I. Abe, T. Watanabe, W. Lou and H. Noguchi, FEBS J., 2006, 273, 208-218.

60 E. A. Ferreira, E. F. Gris, J. M. Rebello, J. F. G. Correia, L. F. S. de Oliveira, D. Wilhelm and R. C. Pedrosa, Planta Med., 2011, 77, 1569-1574.

61 S. B. Wu, C. Long and E. J. Kennelly, Nat. Prod. Rep., 2014, 31, 1158-1174.
62 C. Blanchard, D. Pouchain, P. Vanderkam, M. C. PeraultPochat, R. Boussageon and H. Vaillant-Roussel, Eur. J. Clin. Pharmacol., 2018, 74, 541-548.

63 M. Bruder, P. L. Haseler, M. Muscarella, W. Lewis and C. J. Moody, J. Org. Chem., 2010, 75, 353-358. 